\title{
CUIDADOS PALIATIVOS: DESAFIOS PARA \\ O ENSINO NA PERCEPÇÃO DE ACADÊMICOS DE ENFERMAGEM E MEDICINA
}

\section{PALLIATIVE CARE: CHALLENGES FOR TEACHING IN THE PERCEPTION OF NURSING AND MEDICAL STUDENTS}

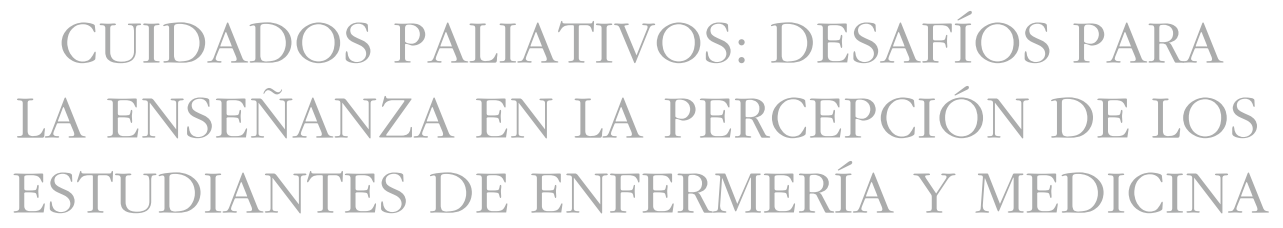

\author{
Ramona Garcia Souza Dominguez \\ Amanda Santos Veiga Freire ${ }^{2}$ \\ Claudia Feio da Maia Lima ${ }^{3}$ \\ Natádina Alves Souza Campos ${ }^{4}$
}

\begin{abstract}
Como citar este artigo: Dominguez RGS, Freire ASV, Lima CFM, Campos NAS. Cuidados paliativos: desafios para o ensino na percepção de acadêmicos de Enfermagem e Medicina. Rev baiana enferm. 2021;35:e38750.

Objetivo: identificar as dificuldades na abordagem dos cuidados paliativos e da terminalidade na percepção de acadêmicos de Enfermagem e Medicina de uma universidade pública. Método: trata-se de estudo exploratório, descritivo, com abordagem qualitativa, cuja coleta de dados foi realizada entre setembro de 2019 e janeiro de 2020, por meio de entrevista semiestruturada. Resultados: emergiram duas categorias temáticas principais: Desafios da comunicação em cuidados paliativos e Despreparo para lidar com o morrer e a morte. Considerações finais: identificou-se que os acadêmicos de Enfermagem e Medicina apresentam dificuldades relacionadas à comunicação na assistência em cuidados paliativos e em situações de fim de vida. Demonstrou-se ainda o seu despreparo em lidar com a morte, o sofrimento humano e os conflitos morais decorrentes de crenças religiosas.
\end{abstract}

Descritores: Cuidado Paliativo. Cuidados de Conforto. Educação de Graduação em Medicina. Educação em Enfermagem. Formação Profissional em Saúde.

Objective: to identify the difficulties in addressing palliative care and terminality in the perception of Nursing and Medical students from a public university. Method: this is an exploratory, descriptive study with a qualitative approach, whose data collection was carried out between September 2019 and January 2020, through semi-structured interviews. Results: two main thematic categories emerged: Challenges of communication in palliative care and Unpreparedness to deal with dying and death. Final thoughts: Nursing and Medical students present difficulties related to communication in palliative care and end-of-life situations. This unpreparedness was also present in dealing with death, buman suffering and moral conflicts arising from religious beliefs.

Descriptors: Palliative Care. Comfort Care. Medical Graduate Education. Nursing Education. Health Professional Training.

\footnotetext{
Enfermeira Oncologista. Doutora em Ciências da Saúde, Epidemiologia do Câncer e Gestão de Unidades Críticas. Professora Adjunta na Universidade Federal do Recôncavo da Bahia. Santo Antônio de Jesus, Bahia, Brasil. ramonagarcia@ufrb.edu.br. http://orcid.org/0000-0002-3131-3953.

Bacharela em Saúde. Universidade Federal do Recôncavo da Bahia. Santo Antônio de Jesus, Bahia, Brasil. http://orcid.org/0000-0003-4525-2490.

Enfermeira. Doutora em Enfermagem. Professora Adjunta na Universidade Federal do Recôncavo da Bahia. Santo Antônio de Jesus, Bahia, Brasil. http://orcid. org/0000-0002-47| 8-8683.

Bacharela em Saúde. Universidade Federal do Recôncavo da Bahia. Santo Antônio de Jesus, Bahia, Brasil. http://orcid.org/0000-000 I-9970-2405.
} 
Objetivo: identificar las dificultades para abordar los cuidados paliativos y la terminalidade en la percepción de los estudiantes de Enfermería y Medicina de una universidad pública. Método: se trata de un estudio exploratorio y descriptivo con un enfoque cualitativo, cuya recogida de datos se llevó a cabo entre septiembre de 2019 y enero de 2020, a través de entrevistas semiestructuradas. Resultados: surgieron dos categorías temáticas principales: Desafios de la comunicación en los cuidados paliativos y Falta de preparación para lidiar con el morir y la muerte. Consideraciones finales: se identificó que los estudiantes de Enfermería y Medicina presentan dificultades relacionadas con la comunicación en cuidados paliativos y situaciones al final de la vida. También se demostró su falta de preparación al tratar la muerte, el sufrimiento humano y los conflictos morales derivados de las creencias religiosas.

Descriptores: Cuidados Paliativos. Cuidados de Confort. Educación de Grado en Medicina. Educación de Enfermería. Formación Profesional en Salud.

\section{Introdução}

A Organização Mundial de Saúde (OMS) define os Cuidados Paliativos (CP) como uma abordagem que objetiva a melhoria da qualidade de vida de pacientes e seus familiares em face aos problemas relacionados às doenças que ameaçam a continuidade de vida, pela prevenção e o alívio do sofrimento, com identificação, avaliação e tratamento precoces da dor e de outras demandas de ordens psicológicas, psicossociais e espirituais ${ }^{(1)}$.

Diante do conjunto de transformações epidemiológicas e demográficas que culminaram no envelhecimento populacional e no aumento na incidência dos casos de Doenças Crônicas não Transmissíveis (DCNT), responsáveis por aproximadamente $80 \%$ das mortes nas Américas ${ }^{(2)}$, os CP representam uma estratégia necessária para a assistência em saúde. Não obstante a expansão progressiva dessa linha de cuidados nos países desenvolvidos, seu acesso ainda é dificultado nos países em desenvolvimento, como o Brasil ${ }^{(1)}$.

Dentre os principais obstáculos apontados pela OMS para o avanço dos CP está o conhecimento deficiente ou inexistente dos princípios e das práticas por profissionais da saúde ${ }^{(1)}$, revelando as lacunas na abordagem dessa temática nas grades curriculares dos cursos de graduação.

Observa-se ainda que a finitude da vida é encarada como sinônimo de falha ou fracasso por profissionais da saúde, sentimento que provém de um processo formativo de valorização dos fundamentos técnicos, em detrimento da concepção holística do ser humano e centrado, quase que exclusivamente, na luta contra a doença, enquanto existem censuras para falar sobre morte. Fatores que resultam na desvalorização de ações que propõem dignidade na terminalidade ${ }^{(3-4)}$ e acarretam desafios emocionais e sensação de despreparo para os CP.

No Brasil, as grades curriculares de Enfermagem apresentam alguns avanços pontuais, como a existência de disciplinas eletivas, obrigatórias e projetos de extensão voltados ao tema ${ }^{(5)}$. Todavia, observa-se que, muitas vezes, a abordagem dos conteúdos relacionados aos CP ocorre de maneira insuficiente no processo formativo de enfermeiros e médicos, podendo culminar em dificuldades emocionais e sentimento de despreparo no cuidado ${ }^{(5-6)}$.

Esta pesquisa justifica-se pela relevância de compreender os desafios para o ensino de CP na graduação em Enfermagem e Medicina sob o olhar dos acadêmicos, a fim de levantar as demandas para a abordagem dessa temática. Busca-se oferecer subsídios para a reflexão sobre as competências do egresso, entendendo que a formação desses profissionais deve estar adequada à realidade epidemiológica do país.

Com base nesse panorama e tendo em vista a atual relevância dessa temática, o estudo objetiva identificar as dificuldades na abordagem dos CP e da terminalidade na percepção de acadêmicos de Enfermagem e Medicina de uma universidade pública.

\section{Método}

Trata-se de estudo exploratório, descritivo, com abordagem qualitativa, norteada pela ferramenta Consolidated criteria for reporting 
qualitative research (COREQ). A escolha dessa abordagem decorreu da possibilidade de compreender as infinitas produções humanas que estão inseridas no meio das relações, representações e da intencionalidade, objeto da pesquisa qualitativa que, muito provavelmente, não podem ser traduzidos em números e indicadores quantitativos $^{(7)}$.

Os participantes foram 44 acadêmicos de uma Instituição de Ensino Superior (IES) Federal, sendo 23 discentes de Enfermagem e 21 do curso de Medicina. Utilizou-se como critérios de inclusão ser estudante de Enfermagem ou Medicina, com idade igual ou superior a 18 anos, regularmente matriculado a partir do sexto semestre, uma vez que estes passam a ter contato com os conteúdos dos cursos de saúde de forma mais específica. Destaca-se que o local de estudo adota a modalidade de formação em dois ciclos. Os cursos de Enfermagem e Medicina estão estruturados em 11 e 14 semestres, respectivamente, sendo que os primeiros 6 semestres são cursados no Bacharelado Interdisciplinar em Saúde (BIS). Foram excluídos os estudantes do BIS sem terminalidade definida. Utilizou-se amostragem aleatória para escolha dos participantes, e a coleta de dados foi encerrada por saturação teórica $^{(8)}$, isto é, no momento em que não houveram informações novas sobre o fenômeno estudado.

Realizou-se a coleta de dados entre setembro de 2019 e janeiro de 2020, por meio de entrevista semiestruturada, agendadas por meio de formulário eletrônico enviado por e-mail, realizadas presencialmente em sala reservada na própria Universidade e gravadas com aparelho eletrônico. Utilizou-se roteiro composto por perguntas abertas e fechadas, para possibilitar que o entrevistado discorresse sobre o assunto em questão sem se restringir à questão colocada ${ }^{(7)}$. As variáveis de interesse foram: idade, sexo, curso de graduação, semestre, ano de ingresso e religião. No roteiro da entrevista apresentou-se a questão norteadora: "Quais são as dificuldades que você identifica na abordagem dos CP e da terminalidade durante a sua formação acadêmica?"
Os áudios das entrevistas foram transcritos, confirmados e analisados, seguindo o caminho da análise de conteúdo ${ }^{(9)}$, composta pelas etapas: pré-análise (organização e transcrição do material coletado); exploração do material (leituras sucessivas e extração das ideias centrais expressas, para sistematizar os dados); e construção das categorias e agrupamento dos elementos, segundo suas semelhanças. Do tratamento dos resultados emergiram duas categorias temáticas principais: "Desafios da comunicação em cuidados paliativos" e "Despreparo para lidar com o morrer e a morte."

O estudo foi aprovado pelo Comitê de Ética em Pesquisa (CEP) com seres humanos sob o Parecer n. 2.857.547 e o Certificado de Apresentação para Apreciação Ética (CAAE) n. 95097218.0.0000.0056, e foi conduzido de acordo com os padrões éticos exigidos e respeitando a Resolução n. 510/16, do Conselho Nacional de Saúde (CNS). O anonimato dos participantes foi mantido com a substituição dos nomes por AE (acadêmico de Enfermagem) ou AM (acadêmico de Medicina), seguidos de números que corresponderam à sequência das entrevistas.

\section{Resultados}

Entre os 21 acadêmicos de Medicina, 15 eram do sexo feminino e 6 , do masculino, com idades variando entre 21 e 39 anos. Quanto à religião, os participantes declararam-se protestantes (11), católicos (4), sem religião (4), messiânico (1) e pagão (1). Dentre os religiosos, 9 declararam-se praticantes, 1 não praticante, e 7 não responderam à pergunta. Em relação ao período acadêmico, os participantes cursavam o sexto semestre (4), o sétimo (2), o décimo (3), o décimo primeiro (2), o décimo segundo (1), o último ano, internato, ${ }^{(8)}$ e não forneceu essa informação (1).

Dentre os 23 acadêmicos de Enfermagem, 20 eram do sexo feminino e 3 do masculino, com faixa etária entre 19 e 51 anos. Quanto à religião, 7 eram protestantes, 11 católicos, 3 espíritas e 2 sem religião. Dos religiosos, 11 eram praticantes, 6 não praticantes e 4 não responderam 
à pergunta. Quanto ao período acadêmico, 9 participantes cursavam o sexto semestre; 12 , o sétimo; 1, o oitavo; e 3, o nono.

Após a análise dos resultados, emergiram duas categorias, que estão descritas a seguir: Desafios da comunicação em cuidados paliativos e Despreparo para lidar com o morrer e a morte:

\section{Desafios da comunicação em cuidados paliativos}

Esta categoria traduz a ideia que os participantes têm acerca dos obstáculos na comunicação, para abordagem da equipe junto a pacientes e familiares, quando o plano terapêutico está pautado em CP. As falas dos entrevistados evidenciaram desafios no processo de comunicação em CP, principalmente nas relações dos profissionais com os pacientes em paliação e seus familiares. Evidenciou-se o despreparo e a insegurança para o diálogo com esses indivíduos, como a dificuldade para lidar com as suas subjetividades:

\begin{abstract}
As principais dificuldades que eu encontro, eu acho que são relacionadas mais a forma de lidar mesmo com o paciente e com a família, de conversar sobre essas questões, porque ainda é muito pouco discutido na universidade. (AE19).
\end{abstract}

Ainda falta muita coisa para aprender, consolidar esse conbecimento e poder lidar com o processo de terminalidade, tanto em questão com o paciente quanto com os familiares, como abordar, fazer essa abordagem nessa situação da terminalidade. Além do conbecimento em algumas coisas, como falar, como transmitir essas informações, falar para a família ou para o paciente que esse já é um estado terminal, o saber mesmo se comunicar. (AE22).

Então, eu acho que essa é a parte mais dificil de tudo, é dificil pra mim conversar com uma familia que haveria ali a possibilidade de ser um cuidado paliativo e de fazer essa família entender o que é um cuidado paliativo. Pra mim essa é a grande dificuldade. (AM15).

Alguns participantes revelaram que se sentiram humanamente conectados para lidar com o outro, numa perspectiva relacional envolvendo a comunicação e o relacionamento interpessoal, por acreditarem ser relevante; contudo, identificam a necessidade de associação com uma formação técnico-científica consistente sobre CP:

O que eu tenho a oferecer no momento são babilidades bumanísticas de cuidado, sensibilidade, coisa que já considero bastante relevante para os cuidados paliativos. Mas necessito, para além das habilidades que possuo, de conbecimentos técnico-científicos, que me ofereçam estratégias para a promoção de um cuidado integral mais possivel a esses pacientes. (AE23).

Destaca-se que a ineficiência da comunicação interprofissional foi extraída das falas dos entrevistados do curso de Medicina, revelando dificuldades quanto à compreensão e o cumprimento do plano de cuidados do paciente pela equipe, questão não citada pelos discentes de Enfermagem:

[...] tem que ter uma comunicação muito grande da equipe para gente determinar que aquele paciente é um paciente paliativo. E na passagem de plantão para o outro, às vezes, não é informado que o paciente é paliativo. Aí vai e colocam determinadas medicações, antibiótico, em pacientes que são cuidados paliativos, que já não são mais [...] não é para realizar mais esse cuidado. (AM3).

[...] a maior dificuldade é justamente dos outros profissionais, porque a partir do momento que a gente tem entendimento como médico de que há uma finitude do tratamento, a equipe entender isso também é complicado. Durante o internato teve um caso desses na cirurgia, já tinha todo um provável desfecho negativo e decidiu-se encerrar os esforços, dar mais conforto pra ele até chegar a morte, só que a equipe ficou bem ouriçada em relação a isso, porque eles interpretavam que havia mais coisa para fazer. Por que não faz isso? [...] Não vai mudar em nada o desfecho do paciente [...] não faz sentido. É pouco discutido e a equipe não tem tanta profundidade nas discussões sobre paliação e finitude. (AM20).

\section{Despreparo para lidar com o morrer e a} morte

Esta categoria evidencia a inaptidão que os participantes expressam diante da realidade do morrer e a morte, pelo sentimento de muita vulnerabilidade, pela dificuldade de aceitação da finitude e da perspectiva maior de saúde para curar e não cuidar. As unidades de significados revelaram a existência de lacunas diante da terminalidade e o processo de morte e morrer. Suas falas destacaram o despreparo e a insegurança para noticiar e lidar com o desfecho de morte:

Eu acho que [a dificuldade é] a falta de preparo que a gente tem sobre cuidados paliativos e acaba dificultando nossa reação diante da situação. Eu acho que no caso de dar a notícia de morte seria o mais complicado. (AE5).

Eu acho que uma dificuldade pra mim, assim, seria lidar com o momento da morte, com a família, eu não sei como seria minha reação no serviço, não tenbo esse preparo na prática, realmente, em vivenciar. (AE17). 
Acho que pra mim [a dificuldade] seria muito mais a questão de lidar com o processo de morte, com aceitar esse processo de morte, com o sofrimento do familiar, acompanhar essa família, esse paciente e dentro de mim entender como se deu esse processo de morte, aceitar [...]. (AE18).

Ainda foram demonstrados os desafios enfrentados pelos acadêmicos dos dois cursos para a desconstrução da ideia negativa de morte, como o despreparo emocional para o enfrentamento das questões referentes à terminalidade:

\begin{abstract}
A morte ainda é tratada como um tabu, ainda se tenta de todas as formas evitá-la, mesmo que isso traga mais sofrimento ao individuo. Falar em cuidado paliativo remete a morte e é dificil discutir sobre isso. (AM6).

Desconstruir toda ideia de morte como algo que traz apenas dor, de falha profissional para a ideia de finalização de um ciclo. (AE9).

[...] as dificuldades encontradas são mais no quesito em como eu vou lidar com essa situação, pelo fato de que eu sou muito sensivel e acabar me envolvendo muito emocionalmente com a dor do outro. (AE12).
\end{abstract}

Para além dos pontos já destacados, os acadêmicos do curso de Medicina citaram importantes questões no que se refere aos conflitos morais de condutas no processo de identificação e definição do momento de terminalidade, além dos entraves encontrados para desassociar as crenças religiosas de aspectos do cuidado:

\begin{abstract}
Já participei de uma extubação paliativa que foi o que me deu o alerta de que não estava tudo tão bem resolvido assim como eu imaginava [...]. Para mim, ia ser muito tranquilo, mas depois os meus julgamentos morais ficaram recobrando isso, se o que a gente tinha feito era antecipar a morte da paciente ou se o que a gente tinha feito era confortar, dar, permitir uma morte confortável. (AM4).

Minha dificuldade é entender, é separar a fé do real, porque como eu ten bo fé, é que sou cristã então, eu acredito que Deus pode fazer o impossível, por exemplo. Acho que é essa minha maior dificuldade. Já teve momentos de eu questionar: será que eu não poderia ter tido um pouco mais de fé nesse paciente? Se demorasse mais um pouco talvez ele não conseguisse voltar assim como outro paciente voltou? (AM5).
\end{abstract}

\section{Discussão}

Os achados desta investigação indicam que o conhecimento acerca da morte e do morrer, realidade próxima a muitos pacientes em $\mathrm{CP}$, ainda é ínfimo na perspectiva da abordagem e do aprofundamento na formação profissional de enfermeiros e médicos, com destaque para as competências de comunicação interpessoal e em equipe para dar más notícias, e a naturalização do processo de morte e morrer. Os graduandos de ambos os cursos apresentaram dificuldades para a consolidação da comunicação como instrumento do cuidado nas práticas de saúde, sobremaneira na condição de CP e/ou terminalidade.

A comunicação faz parte das relações humanas e é uma habilidade essencial da atenção à saúde. Os acadêmicos trazem à tona as dificuldades no diálogo com pacientes e familiares em situações críticas e de fragilidade. Esse resultado foi encontrado em estudo desenvolvido no laboratório de práticas de enfermagem de um curso de graduação no sul do Brasil, quando da simulação realística por meio de encenação de atores voltada à comunicação do diagnóstico de câncer e início dos CP, e observou-se os participantes quanto ao comportamento diante da comunicação de situação crítica em $\mathrm{CP}^{(10)}$.

Os dados resultaram na identificação do medo de falar com a família, a insegurança de passar por essa realidade, a pouca vivência nessas situações e os sentimentos de negação, raiva e culpa. O uso da simulação realística permitiu o desenvolvimento da escuta ativa, empatia e comunicação não verbal, enquanto habilidades e competências a serem aprimoradas na graduação. Esse processo pode ainda auxiliar os acadêmicos, enquanto método de aprendizado, como facilitador do diálogo acerca do processo da morte e do morrer, qualificar a comunicação de notícias difíceis que demandam sutileza e delicadeza na sua abordagem e compreensão dos próprios sentimentos, no tocante ao cuidado em saúde ${ }^{(10)}$.

Essas dificuldades ficam mais evidentes quando se resgata o predomínio histórico da verticalização do cuidado e da hegemonia médica que permeiam a relação equipe-paciente e da construção de uma atenção em saúde fragmentada e focada na doença ${ }^{(11)}$.

Entretanto, por possuir caráter multidisciplinar e assistência voltada à manutenção integral da qualidade de vida dos pacientes fora de possibilidade terapêutica e seus familiares, 
os CP possibilitam o resgate e a revalorização dos relacionamentos interpessoais no processo de cuidar, até mesmo após a morte do paciente. Logo, a comunicação apresenta-se como ferramenta essencial para o cuidado em saúde, por possibilitar a identificação e o acolhimento das necessidades dos pacientes em paliação ${ }^{(12-13)}$ e o preparo dos familiares para a vivência do processo de luto ${ }^{(14)}$, reforçando o caráter humano do cuidar.

Ademais, a comunicação sobre a assistência de fim de vida com os indivíduos em processo de morte e morrer viabiliza, muitas vezes, uma relação de maior concordância quanto ao plano de cuidados entre o profissional e o paciente, impactando a tomada de decisões referente às intervenções a serem realizadas ${ }^{(15)}$. Os resultados de um estudo americano revelaram que discutir as questões de cuidado em fim de vida, pelo menos 30 dias antes das mortes dos pacientes assistidos, permitiu que eles estivessem mais receptivos aos $\mathrm{CP}$ e menos propensos às intervenções agressivas, acarretando em melhor qualidade no momento da partida ${ }^{(16)}$.

Os achados desta pesquisa demonstraram a importância de formar profissionais capazes de lidar com as demandas biopsicossociais e espirituais, pelo desenvolvimento de habilidades humanas individuais, ao tempo em que destacaram lacunas nos conteúdos teóricos. Por si só, ensinar o que não se pode tornar técnico já é um grande desafio que demanda a transversalidade do tema na matriz curricular dos cursos de graduação, para propiciar vivências práticas no lidar com a subjetividade do ser. Ainda que essas experiências tragam resultados plurais, pelo processo natural de amadurecimento profissional, oferecem a oportunidade de serem trabalhados aspectos individuais, para alicerçar maior confiança ao futuro profissional ${ }^{(17)}$.

Outra pesquisa demonstrou que os discentes de Enfermagem e Medicina reconheceram os estágios e práticas como oportunidade para o desenvolvimento das habilidades necessárias para se prestar esse tipo de cuidado. Essa estratégia de ensino permitiu exercitar a busca de equilíbrio emocional em meio ao processo de autoidentificação ou identificação familiar com as situações vivenciadas pelos pacientes, iniciando um ciclo de empatia e distanciamento oriundo da maturidade emocional necessária ao paliativismo $^{(17)}$.

Evidenciou-se também que a falta de componentes curriculares obrigatórios que abordem $\mathrm{CP}$ e terminalidade de maneira transversal gera dificuldades de aprendizagem nas práticas e estágios, pois o discente não foi apresentado ao conteúdo teórico. A oferta de disciplinas optativas pode suplementar essa defasagem, mas não deve ser a estratégia principal para preparar os discentes para lidarem com essas situações, garantindo o direito à dignidade e o conforto em final de vida aos pacientes assistidos ${ }^{(17)}$.

Um estudo realizado em diversos hospitais com médicos residentes de diferentes especialidades e acadêmicos de Medicina de uma instituição privada de ensino mostrou que apenas $23 \%$ dos participantes avaliaram suas habilidades comunicacionais como boas ou muito boas, e falar sobre o fim das tentativas de tratamento curativo foi apontada como a notícia mais difícil de ser comunicada. Mais de 50\% desses sujeitos sentiram-se impactados pela comunicação da má notícia, relacionando-a a questões emocionais e de baixa autoestima profissional ${ }^{(18)}$.

Nos CP, a forma como uma mensagem é transmitida influencia a maneira como o paciente e os familiares lidarão com o diagnóstico, o tratamento e o momento de fim de vida ${ }^{(19)}$. Isso reforça o entendimento de que o desenvolvimento dessas habilidades comunicacionais deve ocorrer durante a graduação, para ampliar o olhar dos acadêmicos sobre a complexidade assistencial desses indivíduos, respeitando a autonomia para com seu plano de cuidados e o fortalecimento do vínculo com a equipe, proporcionando confiança, tranquilidade e conforto ${ }^{(12-13)}$.

O trabalho multidisciplinar e a comunicação na equipe compreendem outros grandes desafios para o ensino de CP, pela compreensão da importância da superação de empecilhos existentes na comunicação e no relacionamento interprofissional, bem como pela relevância no alcance de uma assistência bem sucedida em CP. 
Esse modelo de trabalho exige dos profissionais a capacidade de diálogo com outras áreas do conhecimento, flexibilidade, respeito, valorização dos demais saberes e liderança compartilhada ${ }^{(20)}$.

A associação desses fatores é fundamental para a definição do plano de cuidados adequado ao paciente em paliação e atenção à família, com respeito aos princípios da beneficência e não maleficência. Outras demandas importantes para o ensino revelaram-se nos achados referentes aos desafios enfrentados diante do desfecho de morte que perpassam a sua aceitação, o lidar com o sofrimento humano, os conflitos decorrentes de crenças religiosas e as intervenções para a qualidade no fim de vida.

No cenário sociocultural ocidental contemporâneo, a morte configura um dos principais tabus, descrita como algo indesejável, desagradável e mal compreendida ${ }^{(21)}$. Esses fatores podem ser reforçados por uma formação tecnicista, focada no curar ao invés de cuidar, gerando para os acadêmicos sentimento de rejeição da temática, que considera a prática de ensino ainda muito resistente à morte, capaz de contribuir para $\mathrm{O}$ despreparo na condução da assistência no fim de vida.

Esses aspectos demonstram que a formação acadêmica pautada na valorização da preservação e manutenção da vida a qualquer custo ${ }^{(22)}$, em detrimento do cuidado com foco na qualidade e nas questões que permeiam a morte, o morrer e o luto enquanto eventos naturais, podem contribuir para a associação da morte com falha profissional, insucesso e dilemas emocionais.

Estudo realizado com profissionais e acadêmicos das áreas de Medicina, Enfermagem e Terapia Ocupacional demonstrou que essas categorias não se sentiam preparadas pela universidade para o enfrentamento da morte, em virtude da abordagem superficial do tema ${ }^{(21)}$. Esse fato pode ser explicado, em parte, pelo próprio despreparo do docente em tratar questões da terminalidade, devido a suas crenças e valores pessoais; reflexo também de uma formação que precisa ser revista, atualizada e aprimorada continuamente.
Ademais, esse tema tem sido comumente abordado de forma isolada em disciplinas não obrigatórias específicas do currículo e com conteúdo excessivamente teórico, e as experiências reais durante a graduação com a morte de pacientes são tratadas com distanciamento e silêncio. Entende-se que a morte é um dos possíveis desfechos da assistência à saúde em todos os ciclos de vida, o que torna necessário o investimento na capacitação do docente para ensinar os cuidados de fim de vida com abordagens mais amplas e transdisciplinares em diferentes momentos e oportunidades da formação médica e de enfermagem, inclusive nas práticas e estágios ${ }^{(23)}$.

A abordagem desses temas ainda na graduação permite identificar sentimentos e atitudes conflitantes nos acadêmicos, que podem ser discutidos e trabalhados mediante um olhar docente sensível e humano. No que tange à equipe de Enfermagem em ambiente hospitalar, acerca dos desafios e conflitos vivenciados junto ao paciente em processo de morte/morrer, os sentimentos mais recorrentes foram de tristeza, dor, empatia e consternação ${ }^{(24)}$, corroborando os resultados deste estudo quanto às demandas emocionais encontradas no enfrentamento da terminalidade e morte.

Ademais, durante o cuidado ao paciente em finitude, os profissionais enfrentam uma série de divergências referentes às condutas terapêuticas, sendo citada a extubação paliativa por um dos participantes do estudo. Assim, questionamentos morais e éticos inquietam a equipe diante da assistência em CP, já que intervenções que retardam a partida não são mais prioritárias ${ }^{(24)}$, pois buscam oferecer conforto ao paciente, evitando intervenções desnecessárias e que não apresentam resultados terapêuticos. Tais questionamentos refletem a valorização da manutenção da vida a qualquer custo, mesmo com sofrimento, em vez de focar na boa morte. São fatores que evidenciam o despreparo e o conhecimento insuficiente para a condução conjunta de um plano terapêutico paliativo.

As crenças religiosas fazem parte das estratégias utilizadas pelos acadêmicos de saúde para 
o enfrentamento das dificuldades com a morte e o morrer, como demonstrado no estudo realizado em um hospital universitário com discentes de Enfermagem ${ }^{(22)}$. Todavia, ao fazer uso dessa estratégia, o profissional precisa encontrar equilíbrio na tomada de decisões, uma vez que essas situações podem gerar conflitos internos secundários devido a limitações das intervenções assistenciais diante da autonomia do paciente e da inexorabilidade da morte. Nesse sentido, é de suma importância a inserção de forma ampliada e permanente dessas temáticas no processo formativo desses profissionais,

Este estudo contribuiu para a visibilidade das lacunas na formação profissional de médicos e enfermeiros na abordagem dos $\mathrm{CP}$, terminalidade e morte, propiciando reflexões para o entendimento da inclusão e/ou do aprimoramento necessários nas grades curriculares desses cursos de graduação, para a formação profissional em saúde mais ampla, qualificada e segura. A coleta de dados em apenas uma Instituição de Ensino Superior pode ser apontada como limitação deste estudo.

\section{Considerações Finais}

Identificou-se que os participantes desta pesquisa apresentam dificuldades convergentes, relativas à comunicação de temas comuns em $\mathrm{CP}$, em especial, em situações de fim de vida. Demonstrou-se, igualmente, o despreparo para lidar com a morte e o morrer, o sofrimento humano, a limitação terapêutica e os conflitos morais decorrentes de crenças religiosas. Esses achados apontam para demandas de ensino direcionadas a abordagens em CP nos cursos de graduação em Enfermagem e Medicina.

Olhar para graduandos de cursos diferentes em formação na saúde confirmou o quanto esse cuidado se fragiliza se a comunicação não for efetiva e agregadora. As dificuldades apontadas na pesquisa mostraram-se muito próximas, porque, além de unirem fragilidades na formação, revelam o grande desafio da relação interpessoal eficiente, geradora de segurança e apoio, em destaque para situações que lidam com $\mathrm{CP}$ e o processo de morte e morrer.

\section{Colaborações:}

1 - concepção, projeto, análise e interpretação dos dados: Ramona Garcia Souza Dominguez, Amanda Santos Veiga Freire, Claudia Feio da Maia Lima e Natádina Alves Souza Campos;

2 - redação do artigo e revisão crítica relevante do conteúdo intelectual: Ramona Garcia Souza Dominguez, Amanda Santos Veiga Freire, Claudia Feio da Maia Lima e Natádina Alves Souza Campos;

3 - aprovação final da versão a ser publicada: Ramona Garcia Souza Dominguez, Amanda Santos Veiga Freire, Claudia Feio da Maia Lima e Natádina Alves Souza Campos.

\section{Financiamento:}

Fundação de Amparo à Pesquisa do Estado da Bahia (FAPESB).

\section{Referências}

1. Connor SR, Bermedo MCS, editors. Global Atlas of Palliative Care at the End of Life [Internet]. London: Worldwide Palliative Care Alliance; World Health Organization; 2014 [cited 2020 Apr 10]. Available from: www.who.int/nmh/Global_Atlas_ of_Palliative_Care.pdf

2. Pan-American Health Organization. NCDs at a Glance: NCD Mortality and Risk Factor Prevalence in the Americas [Internet]. Washington, D. C.: PAHO; 2019 [cited 2020 May 2]. Available from: https://iris.paho.org/handle/10665.2/51696

3. Orth LC, Haragushiku EY, Freitas ICS, Hintz MC, Marcon CEM, Teixeira JF. Conhecimento do Acadêmico de Medicina sobre Cuidados Paliativos. Rev bras educ med. 2019;43(Suppl 1):286-95. DOI: 10.1590/1981-5271v43suplemento1-20190039

4. Brito PCC, Sobreiro IZ, Atzingen DANCV, Silva JV, Mendonça ARA. Reflexões sobre a Terminalidade da Vida com Acadêmicos de Medicina. Rev bras educ med. 2020;44(1):e033. DOI: 10.1590/1981-5271v44.1-20190213.ing 
5. Gonçalves RG, Silveira BRD, Pereira WC, Ferreira LB, Queiroz AAR, Menezes RMP. Ensino dos cuidados paliativos na graduação de enfermagem. Rev Rene. 2019;20(1):e39554. DOI: $10.15253 / 2175-6783.20192039554$

6. Lustosa AM, Dutra F, Moreira MADM, Evangelista CB, Duarte MCS, Zaccara AAL, et al. Cuidados Paliativos: discurso de médicos residentes. Rev Med Minas Gerais. 2015;25(3):36974. DOI: $10.5935 / 2238-3182.20150072$

7. Minayo MCS. Ciência, técnica e arte: Desafio da pesquisa social. In: Deslandes SF, Cruz Neto O, Gomes R, Minayo MCS. Pesquisa social: teoria, método e criatividade. Petrópolis (RJ): Vozes; 2016. p. 9-29.

8. Minayo MCS. Amostragem e saturação em pesquisa qualitativa: consensos e controvérsias. Rev Pesq Qualitativa [Internet]. 2017 [cited 2020 Jun 23];5(7):1-12. Available from: https://editora. sepq.org.br/index.php/rpq/article/viewFile/82/59

9. Bardin L. Organização da análise. In: Bardin L. Análise de conteúdo. Lisboa: Edições 70; 2011. p. 95-101.

10. Bellaguarda MLR, Knihs NS, Canever BP, Tholl AD, Alvarez AG, Cunha GT. Simulação realística como ferramenta de ensino na comunicação de situação crítica em cuidados paliativos. Esc Anna Nery. 2020;24(3):e20190271. DOI: 10.1590/2177-9465-ean-2019-0271

11. Coriolano-Marinus MWL, Queiroga BAM, Ruiz-Moreno L, Lima LS. Comunicação nas práticas em saúde: revisão integrativa da literatura. Saúde Soc. 2014;23(4):1356-69. DOI: 10.1590/ S0104-12902014000400019

12. Andrade GB, Pedroso VSM, Weykamp JM, Soares LS, Siqueira HCH, Yasin JCM. Cuidados paliativos e a importância da comunicação entre o enfermeiro e paciente, familiar e cuidador. Rev Fund Care Online. 2019;11(3):713-7. DOI: 10.9789/2175-5361.2019.v11i3.713-717

13. Andrade CG, Costa SFG, Costa ICP, Santos KFOS, Brito FM. Cuidados paliativos e comunicação: estudo com profissionais de saúde do serviço de atenção domiciliar. Rev Fund Care Online. 2017;9(1):215-21. DOI: 10.9789/2175-5361.2017. v9i1.215-221

14. Shirado A, Morita T, Akazawa T, Sato K, Tsuneto S, Shima Y. Both maintaining hope and preparing for death: effects of physicians' and nurses' behaviors from bereaved family members' perspectives.
J Pain Symptom Manage. 2013;45(5):848-58. DOI: 10.1016/j.jpainsymman.2012.05.014

15. Brighton LJ, Bristowe K. Communication in palliative care: talking about the end of life, before the end of life. Postgrad Med J. 2016;92:466-70. DOI: $10.1136 /$ postgradmedj-2015-133368

16. Mack JW, Cronin A, Keating NL, Taback N, Huskamp HA, Malin JL, et al. Associations between end-of-life discussion characteristics and care received near death: a prospective cohort study. J Clin Oncol. 2012;30(35):4387-95. DOI: 10.1200/ JCO.2012.43.6055

17. Costa AP, Poles KS, Silva AE. Formação em cuidados paliativos: experiência de alunos de medicina e enfermagem. Interface (Botucatu). 2016;20(59):1041-52. DOI: 10.1590/ $1807-57622015.0774$

18. Vogel KP, Silva JHG, Ferreira LC, Machado LC. Comunicação de Más Notícias: Ferramenta Essencial na Graduação Médica. Rev bras educ med. 2020;43(Suppl 1):314-21. DOI: http://dx.doi. org/10.1590/1981-5271v43suplemento1-20180264

19. Bastos BR, Fonseca ACG, Pereira AKS, Silva LCS. Formação dos Profissionais de Saúde na Comunicação de Más Notícias em Cuidados Paliativos Oncológicos. Rev Bras Cancerol. 2016;62(3):263-6. DOI: https://doi. org/10.32635/2176-9745.RBC.2016v62n3.342

20. Caldas GHO, Moreira SNT, Vilar MJ. Cuidados paliativos: Uma proposta para o ensino da graduação em Medicina. Rev bras geriatr gerontol. 2018;21(3):261-71. DOI: 10.1590/1981-22562018021.180008

21. Sartori AV, Battistel ALHT. A abordagem da morte na formação de profissionais e acadêmicos da enfermagem, medicina e terapia ocupacional. Cad Bras Ter Ocup. 2017;25(3):497-508. DOI: https:// doi.org/10.4322/2526-8910.ctoAO0770

22. Salum MEG, Kahl C, Cunha KS, Koerich C, Santos TO, Erdmann AL. The process of death and dying: challenges in nursing care for patients and family members. Rev Rene. 2017;18(4):528-35. DOI: https://doi. org/10.15253/2175-6783.2017000400015

23. Marques DT, Oliveira MX, Santos MLG, Silveira RP, Silva RPM. Perceptions, Attitudes, and Teaching about Death and Dying in the Medical School of the Federal University of Acre, Brazil. Rev bras educ med. 2019;43(3):123-33 DOI: $\quad 10.1590 / 1981-52712015$ v43n3rb20180187 ingles 
24. Huber DJ, Salvaro MS, Medeiros IS, Soratto MT. Desafios e conflitos éticos vivenciados pela equipe de enfermagem com paciente em processo de morte e morrer. Inova Saúde [Internet]. 2017 [cited 2020 Apr 30];6(2):50-72. Available from: https://pdfs.semanticscholar.org/9390/895494b5e 1b6d2e6717e765b12bf953536df.pdf?_ga=2.
$79892631.1499178783 .1599839233-165893634$. 1590684436

Recebido: 12 de setembro de 2020

Aprovado: 8 de novembro de 2020

Publicado: 20 de janeiro de 2021

A Revista Baiana de Enfermagem utiliza a Licença Creative Commons - Atribuição-NãoComercial 4.0 Internacional. https://creativecommons.org/licenses/by-nc/4.0/ Este artigo é de acesso aberto distribuído sob os termos da Licença Creative Commons (CC BY-NC). Esta licença permite que outros remixem, adaptem e criem a partir do seu trabalho para fins não comerciais. Embora os novos trabalhos tenham de lhe atribuir o devido crédito e não possam ser usados para fins comerciais, os usuários não têm de licenciar esses trabalhos derivados sob os mesmos termos. 